\title{
A Hybrid Genetic Algorithm with Multi-Parent Crossover in Fuzzy Rule-Based
}

\author{
Kritbodin Phiwhorm and Kanda Runapongsa Saikaew
}

\begin{abstract}
The fuzzy system has been widely used in several application fields and successfully performed by applying evolutionary. Genetic algorithm (GA) is one of the evolutionary methods for solving optimization problems. The success of GA depends on the design of its search operation which crossover and mutation are important operators to find a promising solution for difficult optimization problems. This article proposes a hybrid genetic algorithm with multi-parent crossover operators (HGA-MC) in fuzzy rule-based. An HGA-MC is used to optimize the fuzzy rule-based of linguistic values, which are associated with the global search. In experiments, the proposed algorithm and other existing algorithms were evaluated using optimization problems in UCI five datasets with different dimensionality. The experimental results showed that the proposed (fuzzy HGA-MC) achieved higher target precision than other existing methods by about $94.31 \%$. Based on experimental results, HGA-MC could search for combinations of the crossover and mutation operators to discover accurate and concise optimization rules than other existing algorithms.
\end{abstract}

Index Terms-Hybrid genetic algorithm, multi-parent crossovers, fuzzy rule-based.

\section{INTRODUCTION}

The fuzzy system has been widely used in several application fields and successfully performed by applying evolutionary methods. Genetic algorithm (GA) is one of the evolutionary methods for solving optimization problems. The success of GA depends on the design of its search operation, which crossover and mutation are an important operator, as well as their proper integration. The traditional approach with random initial population may contain an infeasible solution, which causes GA take longer to converge.

A fuzzy system is an expert system which can process the fuzzy information using a collection of fuzzy membership functions and rules [1]. The rule base consists of a set of fuzzy if-then rules in the form of "IF a set of conditions is satisfied, THEN a set of consequences can be inferred." The rules in a fuzzy system are usually of a form similar to the following:

Rule: If $\mathrm{A}$ is small and $\mathrm{B}$ is large, then $\mathrm{C}$ is medium

where, $\mathrm{A}$ and $\mathrm{B}$ are input variables (data values), and $\mathrm{C}$ is output variable (class), "small" is a membership function defined on A, "large" is a membership function defined on B and "medium" is a membership function defined on $\mathrm{C}$.

In contrast, fuzzy sets and fuzzy logic have proven to be able to transfer human knowledge into manageable computer

Manuscript received August 25, 2017; revised October 17, 2017.

The authors are with Khon Kaen University, Thailand (e-mail: kritbodin@gmail.com). models using linguistic terms. Fuzzy inference systems use some if-then rules to perform nonlinear mappings from input space to output space. Fuzzy rules are obtained from knowledge extracted from experts or extracted from real-world datasets.

The fuzzy system provides a mathematical framework to deal with the uncertainty and vagueness inherently contained in the information used as input data [1]. Inferred knowledge is represented using a set of highly interpretable rule-based and is expressed regarding linguistic variables [2], [3]. Computational artificial intelligence technique used for solving complex optimization problems. Although the evolutionary algorithms family contains some different algorithms, GA is the most popular and widely used in practice [4]-[6]. GA can mark near-optimal solutions and adopt well for complex search spaces with high robust search capabilities [7].

GA uses crossover and mutation as the main search operators. A number of multi-parent crossovers, such as a unimodal distribution crossover (UNDX) [8], simplex crossover (SPX) [9], parent-centric crossover (PCX) [10], and triangular crossover (TC) [11], have also been employed for solving optimization problems. In [12], UNDX could not generate offspring in some cases such as when the population size was too small. SPX could not perform the function that consisted of tightly linked subfunctions. PCX took an excessive amount of time in comparison to other crossover operators. TC worked well where the optimal solution lies on the boundary of the feasible region.

Because of the problems as described, there is still the need to develop algorithms to analyze more accurate optimization rules. Therefore, this article proposes a hybrid genetic algorithm with multi-parent crossover operators (HGA-MC) in fuzzy rule-based. An HGA-MC is used to optimize the fuzzy rule-based of linguistic values, which associated with the global search. There are two key steps in building this model. First, fuzzy rules are generated for optimization. Second, fuzzy rules are obtained to optimize the solution by the HGA approach with multi-parent crossovers.

The rest of the article is organized as follows. Section II presents the related works. Section III describes the proposed model and the design of our algorithm, its similarities and differences to other evolutionary. Experimental results are present in Section IV. Finally, the conclusions are given in Section V.

\section{RELATED WORK}

\section{A. Hybrid Genetic Algorithms}

One important challenge of an HGA (also called memetic 
algorithm) is the tradeoff between global and local searching as it is the case that the cost of global search can be rather high. The local search method is used to locate global turning toward the optimal solutions.

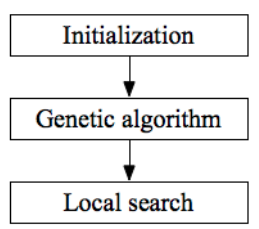

(a) Pre-processor

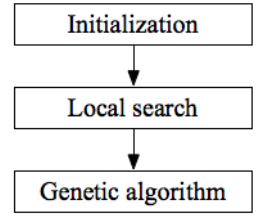

(b) Post-processor

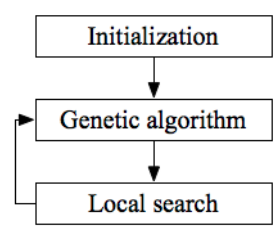

(c) Staged
Fig. 1. Structure of Pipelined Hybrids (a) Pre-processor Hybrids (b) Post-processor Hybrids (c) Staged Hybrids.

In Fig. 1, Sinha and Goldberg [4], presented structural differences of three types of Pipelined Hybrids: (a) Pre-processor Hybrid: the steps of GA techniques to create new populations, then forward the data to the secondary method to find the most appropriate population. (b) Post-processor Hybrids: the secondary method was used to find valuable information appropriately, then send the data to GA techniques to generate new population data. Finally, (c) Staged Hybrids: a loop to get the correct answer from the GA, then forward to the secondary method to search, which then loops back to the GA technique until the most appropriate population is found.

This article chooses to employ Staged Hybrids structure to obtain the most appropriate population as Sinha and Goldberg [4] had verified. In the next section, the proposed model will be presented.

TABLE II: EXAMPLES OF IRIS RULES

\begin{tabular}{cccccc}
\hline \hline $\begin{array}{l}\text { No. of } \\
\text { rule }\end{array}$ & SL & SW & PL & PW & Class \\
\hline 1 & small & large & small & small & Setosa \\
2 & small & medium & small & small & Setosa \\
3 & medium & large & small & small & Setosa \\
4 & small & large & small & small & Setosa \\
5 & small & large & small & small & Setosa \\
6 & large & medium & medium & medium & Versicolour \\
7 & large & medium & medium & medium & Versicolour \\
8 & medium & medium & medium & medium & Versicolour \\
9 & medium & medium & medium & medium & Versicolour \\
10 & large & medium & medium & medium & Versicolour \\
11 & medium & large & large & large & Virginica \\
12 & large & medium & large & large & Virginica \\
13 & medium & medium & medium & large & Virginica \\
14 & large & medium & large & large & Virginica \\
15 & large & medium & large & medium & Virginica \\
\hline \hline
\end{tabular}

\section{PROPOSAL MODEL}

Our proposed fuzzy HGA-MC algorithm is to optimize the fuzzy rule-baed of linguistic values associated with global search.

\section{A. Multi-parent Crossover Operators}

In this research, we deal with real-valued encoding. We propose a multi-parent crossover with the following step:

1) Based on a selection rule, store the individuals that will be used for crossover into a selection pool.

2) Any duplication in the selected three individuals is removed by replacing the unwanted individual with a random individual from the selection pool.
3) Rank these three individuals from the best $\vec{x}_{1}$ to the worst $\vec{x}_{3}$, based on their fitness function.

4) Generate a random number $\beta$.

5) Generate three offspring $\left(o_{i}\right)$.

$$
\begin{gathered}
\vec{o}_{1}=\vec{x}_{1}+\beta\left(\vec{x}_{2}-\vec{x}_{3}\right) \\
\vec{o}_{2}=\vec{x}_{2}+\beta\left(\vec{x}_{3}-\left(\vec{x}_{2}-\vec{x}_{1}\right)\right) \\
\vec{o}_{3}=\vec{x}_{3}+\beta\left(\vec{x}_{1}-\vec{x}_{2}\right)
\end{gathered}
$$

where $f\left(\vec{x}_{1}\right) \leq f\left(\vec{x}_{2}\right) \leq f\left(\vec{x}_{3}\right)$

The idea behind multi-parent crossover comes from the heuristic crossover [13], in which one offspring ( $\vec{O}$ ) is generated from a given pair of parents $\left(\vec{x}_{1}, \vec{x}_{2}\right)$, such that $\vec{o}=\vec{x}_{1}+\beta\left(\vec{x}_{1}-\vec{x}_{2}\right)$, where $\beta$ is a random number between 0 and 1 , and $f\left(\vec{x}_{1}\right) \leq f\left(\vec{x}_{2}\right)$. However, we use three parents instead of two, similarly to the multi-parent crossovers TC, PCX, SPX and UNDX, as discussed earlier. In our case, the difference vectors in the above equations are not in the same order. The order in Eq. 2 is set differently in Eqs. 1 and 3. In fact, Eqs. 1 and 3 are designed to move toward better fitness while Eq. 2 is to diversity the population.

In our case, three offspring are generated from three parents as opposed to one offspring from a pair of parents in heuristic crossover. The differences are:

- For each three distinct parents, three offspring are generated.

- A diversity operator with a probability is used between each generated offspring and different individuals within the archive pool.

- The selection of parents for crossover operation is based on a tournament, and they must be different from each other.

- The best population size individuals from the mix of the archive pool individuals and all generated offspring are selected for the next generation.

\section{B. Optimization of Fuzzy Rule-Based on HGA-MC}

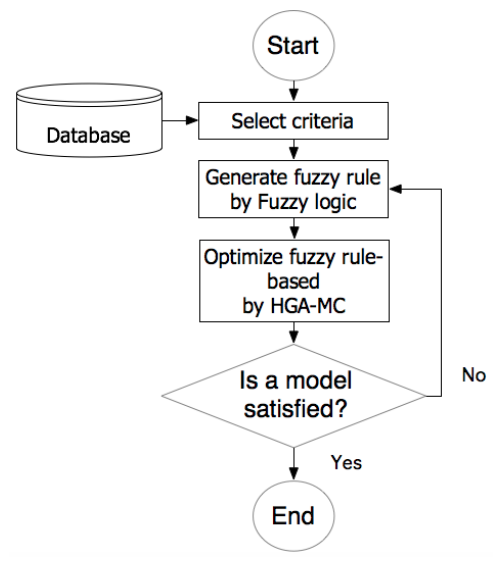

Fig. 2. Overall structure of fuzzy HGA-MC.

Fig. 2 shows the overall structure of fuzzy HGA-MC, and below is a detailed description of framework:

1) A starting select criteria from database. For example, in Table I shows the criteria of Iris data "Sepal length (SL)", "Sepal width (SW)", "Petal length (PL)", "Petal 
width (PW)", "Class"'

2) Next, generate fuzzy rule by using fuzzy logic method. Generate the population of fuzzy rules (chromosomes) by using fuzzy logic method. Fig. 3 shows membership function of Iris data. Examples of Iris membership values are $\{$ small, medium, large $\}$ in Triangular membership function.

For example, data in Table I, and membership function in Fig. 3, can generate fuzzy rule-based as shown in Table II.

1) Next, optimization fuzzy rule-based by fuzzy HGA-MC.

a) Performance of the GA is used to find the appropriate information. Process "selection", "multi-parent crossover" and "mutation" operators determine new population "offspring".

b) Search the fitness value of each chromosome in the population with the local search method which is used as the Staged in the structure of Pipelined Hybrids in Fig. 1.

c) If the value is appropriate or close to zero as the local searcher falls in the local optima.

TABLE I: THE EXAMPLE OF IRIS DATA

\begin{tabular}{llllll}
\hline \hline No. & SL & SW & PL & PW & Class \\
\hline 1 & 4.9 & 3.5 & 1.4 & 0.2 & Setosa \\
2 & 4.9 & 3 & 1.4 & 0.2 & Setosa \\
3 & 5.4 & 3.9 & 1.7 & 0.4 & Setosa \\
4 & 4.6 & 3.4 & 1.4 & 0.3 & Setosa \\
5 & 5 & 3.4 & 1.5 & 0.2 & Setosa \\
6 & 7 & 3.2 & 4.7 & 1.4 & Versicolour \\
7 & 6.4 & 3.2 & 4.5 & 1.5 & Versicolour \\
8 & 6.1 & 2.9 & 4.7 & 1.4 & Versicolour \\
9 & 5.6 & 2.9 & 3.6 & 1.3 & Versicolour \\
10 & 6.7 & 3.1 & 4.4 & 1.4 & Versicolour \\
11 & 6.3 & 3.3 & 6 & 2.5 & Virginica \\
12 & 7.7 & 2.8 & 6.7 & 2 & Virginica \\
13 & 6.3 & 2.7 & 4.9 & 1.8 & Virginica \\
14 & 6.7 & 3.3 & 5.7 & 2.1 & Virginica \\
15 & 7.2 & 3.2 & 6 & 1.8 & Virginica \\
\hline \hline
\end{tabular}

2) Finally, a model is satisfied and stopped with the best population member as the final solution of the fuzzy with HGA-MC process.

To improve the calculation of the complex problems more efficiently and accurately, the local search method embedded in the HGA method, helps to find the correlation coefficients of the independent variables to the target object function.

A multi-parent crossover in genetic algorithm to illustrate the improvement of the proposed method with the new learning procedure. Section IV reports the experimental results of using different three methods for the same set of five different data sets.
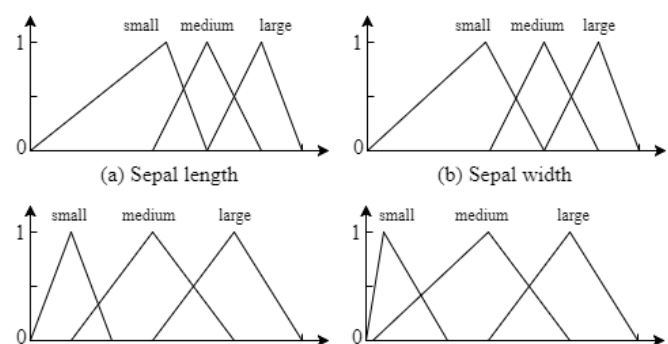

(c) Petal length

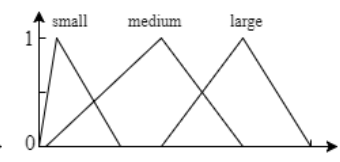

(d) Petal width

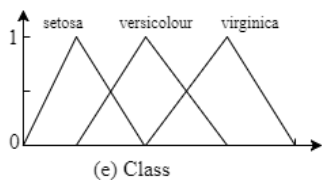

Fig. 3. Sample Triangular membership function of Iris (a) "Sepal length" (b) "Sepal width" (c) "Petal length" (d) "Petal width" (e) "Class".

\section{EXPERIMENTAL RESULTS}

In this section, we show several examples to illustrate the ideas presented in the previous sections. A program using MATLAB programming language was developed to find the optimal values of the results generated by using Fuzzy-GA algorithm, Fuzzy-HGA algorithm and Proposed (Fuzzy-HGA MC) algorithm.

\section{A. Datasets}

This section presents datasets on several well-known real-world benchmarks. The detailed description of these datasets in the University of California Irvine Machine Learning Repository (UCI) [14], including Breast cancer, Diabetes, Glass, Iris, and Liver cancer datasets. These datasets have been used to verify several machine learning algorithms [10].

TABLE III: THE DATASETS OF CHARACTERISTICS AND PARTITIONS

\begin{tabular}{|c|c|c|c|c|c|c|}
\hline \multirow{2}{*}{ Name } & \multirow{2}{*}{ Examples } & \multirow{2}{*}{ Features } & \multirow{2}{*}{ Classes } & \multicolumn{3}{|c|}{ Partition sets } \\
\hline & & & & Training & Validation & Testing \\
\hline Breast cancer & 699 & 9 & 2 & 489 & 210 & 210 \\
\hline Diabetes & 768 & 8 & 2 & 538 & 230 & 230 \\
\hline Glass & 214 & 9 & 6 & 149 & 65 & 65 \\
\hline Iris & 150 & 4 & 3 & 105 & 45 & 45 \\
\hline Liver cancer & 583 & 10 & 2 & 409 & 174 & 174 \\
\hline
\end{tabular}

These characteristics of datasets in Table III shows the diversity in the number of examples, features, and classes. The training set $(70 \%)$ is used to train the algorithm for efficient learning capability, while the testing set $(30 \%)$ is applied to evaluate the generalization ability of the final rule set obtained by the proposed algorithm.

\section{B. Results}

The predictive accuracies (Eq. 4) on the test set of the 10 runs are averaged. (Eq. 5) shows how to calculate the accuracy of measurements.

$$
\begin{aligned}
& \operatorname{accuracy}(\%)=100-\operatorname{error}(\%) \\
& \operatorname{error}(\%)=\left|\frac{\text { Actual }- \text { Measured }}{\text { Actual }}\right| * 100
\end{aligned}
$$

where Actual is the actual value or expected value, Measured is the measured value and error or Relative Error is the difference between Actual and Measured.

The average accuracy results are given in Table IV, which shows the comparison between fuzzy GA algorithm and fuzzy HGA algorithm. Fuzzy GA algorithm, fuzzy HGA algorithm, 
and the proposed fuzzy HGA-MC algorithm are different in local search of HGA. In particular, the proposed fuzzy HGA-MC algorithm included multi-parent crossover operators. From the results, this shows that the incorporation of a local search algorithm with multi-parent crossover achieved higher target precision than other algorithms.

TABLE IV: COMPARISON BETWEEN FUZZY HGA-MC AND OTHER ALGORITHMS ACCURACY RESULTS

\begin{tabular}{lccc}
\hline \multirow{2}{*}{ Name } & \multicolumn{3}{c}{ Average accuracy results (\%) } \\
\cline { 2 - 4 } & Fuzzy GA & Fuzzy HGA & Proposed (fuzzy HGA-MC) \\
\hline Breast cancer & 86.23 & 87.52 & 94.31 \\
Diabetes & 73.96 & 77.31 & 80.27 \\
Glass & 48.16 & 74.16 & 82.62 \\
Iris & 84.00 & 87.34 & 94.55 \\
Liver cancer & 84.49 & 89.09 & 92.53 \\
\hline \hline
\end{tabular}

Based on the experiments using the five UCI datasets with different dimensionality, the proposed (fuzzy HGA-MC) achieved better target precision $(94.31 \%)$ than other methods (fuzzy $\mathrm{GA}=86.23 \%$ and fuzzy $\mathrm{HGA}=87.52 \%$ ). The performances of fuzzy HGA-MC have been compared with the results of other existing well-known optimization algorithms. The computation results, fuzzy HGA-MC can discover more accurate and concise optimization rules than others.

\section{CONCLUSION}

This article proposes a hybrid genetic algorithm with multi-parent crossovers (HGA-MC) to enhance the performance of exploration and exploitation. We also tested different modal functions and analyzed the performance of the convergence of various algorithms. The experimental results of applying GA and HGA algorithm show that the proposed algorithm can improve the ability of global and local searching and overcome premature convergence.

In future, more datasets should be used to evaluate the effectiveness of the proposed algorithm. Also, the diversity in multi-mutation algorithm should be studied to improve local search performance.

\section{ACKNOWLEDGMENT}

This study was supported by Faculty of Engineering, Khon Kaen University, Khon Kaen province, Thailand.

\section{REFERENCES}

[1] J. J. Castro-Schez, J. M. Murillo, R. Miguel, and X. Luo, "Knowledge acquisition based on learning of maximal structure fuzzy rules," Knowledge-Based Syst., vol. 44, pp. 112-120, 2013.

[2] K. Trawiński, O. Cordon, L. Sanchez, and A. Quirin, "A genetic fuzzy linguistic combination method for fuzzy rule-based multiclassifiers," IEEE Trans. Fuzzy Syst., vol. 21, no. 5, pp. 950-965, 2013.
[3] Y. Ding and X. Fu, "Kernel-based fuzzy c-means clustering algorithm based on genetic algorithm," Neurocomputing, vol. 188, no. Supplement C, pp. 233-238, 2016.

[4] D. E. Goldberg, Genetic Algorithms in Search, Optimization and Machine Learning, 1st ed. Boston, MA, USA: Addison-Wesley Longman Publishing Co., Inc., 1989.

[5] K. Phiwhorm and S. Arch-Int, "LDL-cholesterol levels measurement using hybrid genetic algorithm and multiple linear regression," in Proc. 2013 International Conference on Information Science and Applications, ICISA 2013, 2013.

[6] P. Lopez-Garcia, E. Onieva, E. Osaba, A. D. Masegosa, and A. Perallos, "GACE: A meta-heuristic based in the hybridization of genetic algorithms and cross entropy methods for continuous optimization," Expert Syst. Appl., vol. 55, pp. 508-519, 2016.

[7] M. Fazzolari, R. Alcala, Y. Nojima, H. Ishibuchi, and F. Herrera, "A review of the application of multiobjective evolutionary fuzzy systems: Current status and further directions," IEEE Trans. Fuzzy Syst., vol. 21, no. 1, pp. 45-65, 2013.

[8] I. Ono, H. Kita, and S. Kobayashi, "A real-coded genetic algorithm using the unimodal normal distribution crossover," Adv. Evol. Comput., pp. 213-237, 2003.

[9] S. Tsutsui, M. Yamamura, and T. Higuchi, "Multi-parent recombination with simplex crossover in real coded genetic algorithms," in Proc. the 1st Annual Conference on Genetic and Evolutionary Computation, 1999, vol. 1, pp. 657-664.

[10] K. Deb, A. Anand, and D. Joshi, "A computationally efficient evolutionary algorithm for real-parameter optimization," Evol. Comput., vol. 10, no. 4, pp. 371-395, 2002.

[11] E. Z. Elfeky, R. A. Sarker, and D. L. Essam, "Analyzing the simple ranking and selection process for constrained evolutionary optimization," J. Comput. Sci. Technol., vol. 23, no. 1, pp. 19-34, 2008.

[12] S. M. Elsayed, R. A. Sarker, and D. L. Essam, "A new genetic algorithm for solving optimization problems," Eng. Appl. Artif. Intell., vol. 27 , pp. $57-69,2014$

[13] A. H. Wright, "Genetic algorithms for real parameter optimization," Found. Genet. Algorithms, vol. 1, pp. 205-218, 1991.

[14] K. Bache and M. Lichman, "UCI machine learning repository," University of California, Irvine, School of Information and Computer Sciences, 2013.

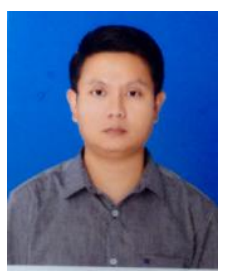

Kritbodin Phiwhorm was born in Amnatcharoen Thailand, in 1978. He received the B.S. degree in computer science from Ramkhamhaeng University, Thailand, and the M.S. in Computer Science from the Khon Kaen University, Thailand, respectively. His current research interests include machine learning, expert system and image processing.

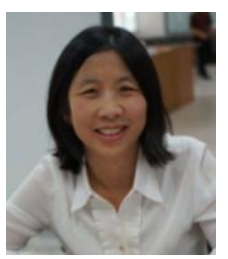

Kanda Runapongsa Saikaew was born in Chiang Rai, Thailand, in 1975. She received the B.S. degree in electrical and computer engineering from Carnegie Mellon University, Pennsylvania, USA, in 1997, and the M.S. and Ph.D. degrees in computer science and engineering from the University of Michigan at Ann Arbor, in 1999 and 2003, respectively. In 2003, she joined the Department of Computer Engineering, Khon Kaen University, as a Lecturer, and became an Associate Professor in 2015. Her current research interests include social network analysis and machine learning. 